\title{
La machine à apprendre
}

Il fut un temps où les abattoirs de la Villette étaient le fournisseur de viande de la ville. Par la suite, on a créé sur les 55 hectares au nord-est de Paris un parc des superlatifs dédié à la technique et aux sciences, à l'art et la culture, aux loisirs et aux divertissements. La Cité des sciences et de l'industrie, la Grande halle, dernier vestige de construction métallique de la cité du sang, et la Cité de la musique, dernière ouvre architecturale en date font partie des fleurons de cette aire de jeu urbaine. Les feuilles mortes de novembre et l'architecture futuriste de la cité se reflètent dans la sphère argentée du cinéma de la Géode qui, grâce à la technologie Imax, transforme les spectateurs en acteurs. «Paris est un bateau magnifique chargé de raison», estimait Balzac. Les présidents de la République l'ont pris au mot et créé, dans les années 80, un chefd'œuvre pédagogique qui, du point de vue didactique, conjugue magnifiquement apprentissage et détente. De nombreuses classes et des familles s'y baladent, mangent dans les nombreux restaurants, partent avec les astronautes de la NASA jusqu'au télescope Hubble, observent les galaxies les plus lointaines, rentrent la tête devant d'énormes dinosaures en 3-D, s'immergent dans l'univers de la science-fiction ou étudient la génétique et l'histoire des sciences. Des consoles d'apprentissage, des bibliothèques et des vidéothèques, ainsi que de nombreux documents multimédias répondent à tous les désirs. Les escaliers roulants véhiculent des milliers de personnes à travers les 5 étages transparents de cette «machine à apprendre» qui dispose également d'un excellent système d'orientation.

Un grand espace d'exposition intitulé Cité de la santé, propose des consultations personnalisées cinq jours par semaine. Un programme mensuel y présente des animations originales, des exposés et des films. «Les vieillards sont-ils des hommes?» demandait Simone de Beauvoir avec un brin de provocation. Quand eston vieux? Que signifie être vieux, chez soi ou en institution? Telles sont les questions que pose une présentation brève avec de beaux portraits de gens âgés. «L'homme est un irréversible incarné», dit la brochure explicative, très informative et pleine d'esprit - comme tout ce qui se fait ici. Et évidemment, on aborde aussi les questions de prévention. Un long panneau fournit une foule d'informations, du sida aux mariages forcés en passant par la nutrition et les 7 règles d'or relatives à l'utilisation de génériques, ainsi que des adresses utiles pour quasiment chaque problème.

La Cité propose une pléthore d'informations. Le flot d'images y est énorme, même là où l'on a expressément le droit de toucher. Des expériences intelli- gentes permettent de comprendre des contenus complexes, car apprentissage doit rimer avec plaisir. Si l'information-divertissement contribue à l'éducation, alors c'est bien ici que cela se passe. Comme toujours, les connaissances préalables et l'approfondissement ultérieurs sont indispensables si l'on ne veut pas rester superficiel. Ce grand bazar du savoir nous donne accès à la compréhension du monde et en demander plus serait irréaliste. Le 150 anniversaire de la théorie de l'évolution de Darwin y a laissé de nombreuses traces. Ce forum d'apprentissage serait en effet fort différent s'il n'avait pas été marqué par ses théories. En 2008, le ministère de l'éducation a organisé une conférence destinée aux enseignants sur le thème «Enseigner l'évolution». Au préalable, de nombreuses écoles et professeurs avaient reçu, sans leur accord, un «Atlas de la Création». Il s'agissait d'un ouvrage illustré pesant plus de 8 kilos qui avait pour but de diffamer la biologie moderne en la qualifiant de tissu de mensonge antireligieux. Cette action en force émanant d'islamistes intégristes turcs et de cercles évangéliques fut un véritable électrochoc de l'avis des participants. La France laïque se découvrait aussi des mouvements fondamentalistes qui cherchent surtout à convaincre les jeunes. Et les jeunes gens professionnellement mal intégrés de rejeter démonstrativement toute discussion rationnelle. Sur internet aussi, les théories de Darwin servent de paratonnerre à la jeunesse désabusée des banlieues et aux adeptes d'un «design intelligent» qui visent à rétablir l'ancienne domination de l'église. La richissime Fondation Templeton met au défi le concept de la Cité des Sciences, car elle préfère subventionner des expéditions de recherche de l'arche de Noé plutôt que le «bateau de la raison» de Balzac. La Cité en devient d'autant plus un lieu de réflexion sociale. Et c'est très bien, car il se peut que dans l'euphorie de sa construction, on ait oublié qu'un projet du siècle des Lumières ne peut pas être un mouvement perpétuel qui continue sa course sans heurt. Les progrès scientifiques ne peuvent perdurer sans prendre en compte la justice sociale et l'écologie. A l'époque de Jules Verne, les changements climatiques, la surpopulation et la biodiversité n'étaient pas encore d'actualité. La Cité lui rend hommage tout en pointant les risques que nous font courir la cupidité, la course au pouvoir et l'exploitation des ressources. Devant la Géode, un groupe de chinoises font du tai-chi. Je leur demande ce qu'elles pensent de la Cité. Elles me répondent en souriant poliment.

Erhard Taverna 